\title{
Real Time Object Detection and Text Extraction
}

\author{
Sunil L., M. Reji
}

\begin{abstract}
- this paper distinguishes and analyzes diverse stages during the time spent content discovery and acknowledgment and investigations distinctive methodologies utilized for content extraction from real time pictures. Two generally utilized techniques for this issue are stepwise strategies and coordinated techniques, though this undertaking is additionally partitioned into content discovery and confinement, grouping, division and content acknowledgment. Imperative methodologies used to experience these stages and their comparing favorable circumstances, disservices and applications are exhibited in this paper. Different content related applications for symbolism are additionally introduced here. This survey performs a similar examination of crucial procedures in this field.

This paper thinks about different stages in the procedure of content discovery and acknowledgment and examinations and analyzes distinctive methodologies used to experience these stages. It presents the significance of each handling stage and focal points, weaknesses, and utilization of methodologies utilized by different supporters of take care of these issues. Different uses of content identification and acknowledgment are additionally assessed in this paper.
\end{abstract}

Content acknowledgment arranges changes over pictures of content into a series of characters or words. It is essential to change over pictures of content into words as a word is a rudimentary substance utilized by a human for his visual acknowledgment. Diverse methodologies of acknowledgment are character acknowledgment and word acknowledgment.

Character acknowledgment techniques separate the content picture into numerous patterns of single characters. The partition between contiguous characters is imperative for these techniques.

Key Words - Detecting Text, Text Recognition, Localization, Classification, Segmentation.

\section{INTRODUCTION}

Object Detection is one of the vital undertakings in video reconnaissance framework. This paper proposes a business related to programmed recognition of surrendered and obscure articles utilizing foundation subtraction, morphological $d$ technique. The point of the methodology is to naturally perceive exercises around confined region to improve security of the adjusting region. The framework takes as contribution from the camera, following and acknowledgment results and fuses these into object estimation. A proposed calculation for item following in video, in light of picture division is proposed. With the picture division all articles in video can be identified

Revised Manuscript Received on September 10, 2019.

Sunil L, UG Scholar, Department of ECE, Saveetha School of Engineering, Saveetha Institute of Medical and Technical Sciences, Chennai, Tamilnadu, India.

(Email: sunilhorror78@gmail.com)

Dr. M. Reji, Assistant Professor, Department of ECE, Saveetha School of Engineering, Saveetha Institute of Medical and Technical Sciences, Chennai, Tamilnadu, India.

(Email: rejime@gmail.com).

whether they are moving or not by utilizing division results of progressive edges. Subsequently, the proposed calculation can be connected to different movements. The calculation was tried on constant video observation framework and it delivers exceptionally low false alerts and missing location. This methodology definitely gives security and recognizes the moving article continuously video arrangement and live video spilling.

Subjective and quantitative outcomes as far as Detection Rate (DR), False Alarm Rate (FAR), achievement rate and assert age handling time per outline are given. The proposed calculations are contrasted and the built up techniques dependent on simple subtraction, pixel values.

\section{Case [1]:}

Late fear based oppressor assaults have featured the requirement for video reconnaissance at open spots. Additionally, early occasion identification is required to maintain a strategic distance from fiascos like flame. Over the most recent two decades, visual reconnaissance has pulled in an ever increasing number of specialists in view of its colossal application prospects. In the greater part of the present setups, the recorded video must be broke down to reproduce an occasion after the related disturbing circumstance has been identified. Due to the successive filtering of the whole video time slip by between the event of occasion and activity required to address the defect increments extensively. Thusly there is a requirement for the advancement of keen reconnaissance framework which will probably naturally identify possibly hazardous circumstances. Specialists are moving in the direction of making the video observation frameworks increasingly flexible, by growing quick, dependable and strong calculations for moving item location, grouping, following, and action examination. Moving images pixel values separation might be complex due to variations in frame rate.

All in all a deserted article is an item which is left at a specific spot under observation and unattended over some undefined time frame. Second, it ought to stay static in ongoing edges or for quite a while t. Identifying a surrendered item is imperative in spots like airplane terminals, railroad stations, enormous shopping centers and so on where there is a possibly high-security risk. AOD is one of the exceedingly difficult errands in video reconnaissance frameworks, a ton of research is done to improve and mechanize the observation framework. 
One of the major and imperative undertakings in a video observation framework is to identify deserted articles. The greatest test in surrendered Object Detection is grouping an item as relinquished article, the item which was absent already in the scene might be a deserted item and for the most part the item turns into a deserted item when it is conveyed by an individual beforehand and it is unattended for a specific timeframe which makes the potential risk.

\section{Case [2]:}

Content location and acknowledgment have developed as an essential issue in the previous couple of years. Progressions in the field of Open CV and AI, just as an expansion in the applications dependent on content identification and acknowledgment, has brought about this pattern. Different workshops and gatherings like the International Conference on Document Analysis and Recognition (ICDAR) are being sorted out on a universal dimension giving further ascent to improvements in the field of content preparing.

Object location and tracking from video frames just as frame per seconds are getting values from algorithms. Enormous work has been done in the field of object identification and extraction from characteristic scenes. Different optical character acknowledgment are accessible. All things considered, the tracking of content location and acknowledgment isn't completely done. Division and extraction of content from regular scenes are still hard to proceed.
This paper thinks about different stages during the object detection and text extraction, content identification and acknowledgment from the taken object in pixel values and looking at changed methodologies used to experience these stages. It introduces the significance of each preparing stage and preferences, weaknesses and uses of methodologies utilized by different supporters of taking care of these issues. Different utilizations of content discovery and acknowledgment are likewise evaluated in this paper.

\section{OBJECT DETECTION AND IDENTIFICATION}

The framework has four primary modules: (a) foregroundextraction; (b) absolute pixel value extraction; (c) Histogram Equalization; (d) CNN location and identification. First back-ground subtraction is performed to identify any new item that may have entered the scene. After that we figure out which individual left sack in the scene. The last module separates among expelled and relinquished items. The framework advises the client of a deserted item by raising an alert.

Containing the input module then transferred to modular resizer unit and with help of Edge detection and Gaussian algorithms identified to differentiate the foreground space and behind space in pixels then output is redirected as object containing image in pixel values and the selection region is applied then data set is compared to every level of recognition and separation are calculated.

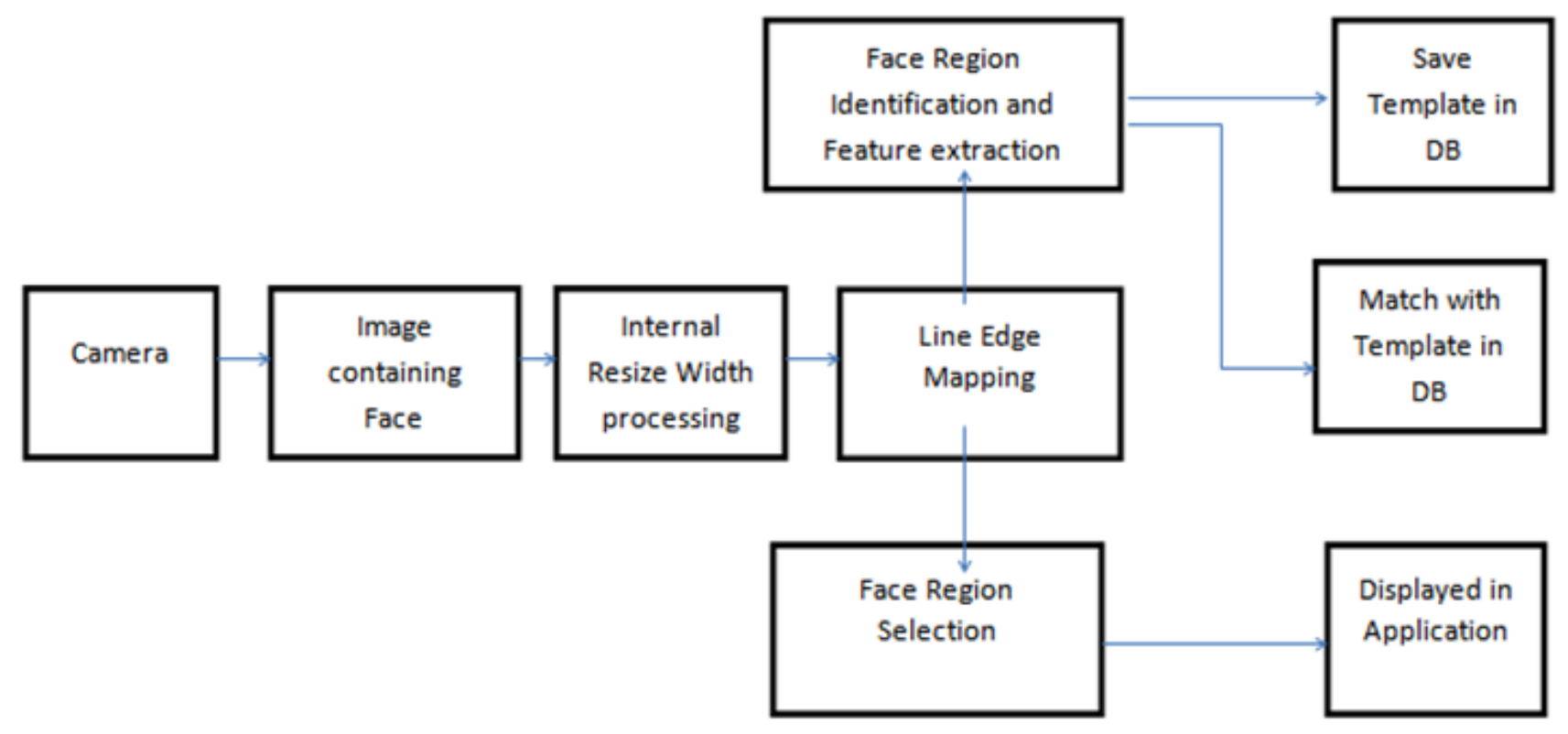

\section{A. Foreground Extraction}

Foregroundextraction is a main piece of object identification frameworks as its output is dependinguponthis procedures, i.e., object acknowledgment and tracking and these procedures depend heavily on the exactness of foregroundextractionalgorithms. This strategy is especially utilized system for segmentation in static camera. It endeavors to recognize moving regions by subtracting the present picture pixel - by-pixel from a reference foundation picture. The pixels where distinction is over an edge are delegated closer view. In the wake of getting a frontal area object, some morphological post handling are performed, for example, expansion and disintegration are performed to lessen the impacts of clamor and improve the identified locales without any loop cases for identification of objects.

\section{B. Absolute pixel value Extraction}

In this technique the absolute and precise method for

Published By: 
extraction and identification of pixel by pixel values emerges. We have to subtract the pixel value information to get threshold value of pixel and this can be used for identification of object that are closer or else farer.

At any given time $t$, the background in the image is given by $\mathrm{V}(\mathrm{x}, \mathrm{y}, \mathrm{t}+1)$, and the reference background image is given byV(x,y, t). When we want to identify the frame difference the method is just to subtract the value in given time i.e., $\mathrm{t}+1$; and therefore $\mathrm{D}(\mathrm{t}+1)=\mid \mathrm{V}(\mathrm{x}, \mathrm{y}, \mathrm{t}+1)-\mathrm{V}(\mathrm{x}, \mathrm{y}$, $\mathrm{t})$ hence the given value according to the statement it said as threshold value of any given image. When an image is moving faster and therefore the threshold value also reaches higher to compensate these variations $\mid V(x, y, t)-V(x, y$, $\mathrm{t}+1) \mid>$ Th.

\section{Equalization of Histogram}

Histogram equalization is a strategy in picture accessingthe differencein change with respect to the original contrast compared utilizing the picture's histogram. This technique builds the images differentiation of numerous pictures, particularly when the usable information of the picture is represented by close complexity esteems and matches accordingly with algorithm. Through this modification, the values can be better organized and extracted through the histogram technique. This takes into account regions of back ground extraction complexity to pick up a higher contrast. Histogram leveling achieves this by successfully spreading out the most values by histogram matrix multiplied to get the images foreground and background isolation. The strategy is valuable in pictures with backgrounds and closer views that are both bright and dim. So in principle, that the histogram equalization capacity is known, extracting the image from the background noise and values are calculated. The calculation isn't computationally escalated. It might build the contrast of background commotion, while diminishing the usable flag foreground.

Let's assume the has the intensity levels variation with respect to frame rate and thus the value 1 is excited for contrast values and value 0 is triggered for contrast values which are barely accepted by histogram.

Contrast value Transformation $S=T(r)=\mid p(w) d w$.

$$
p_{o}(s)=\left\{\begin{array}{l}
1 . \text { For } .0 \leq s \leq 1 \\
0 . \text { Otherwise }
\end{array}\right.
$$

These are values, which the intensity of images are distributed equally with high contrast rate. The system have to done complex variations due to continuous signals from the input module. Since we are working with discrete values the integrate values becomes summation.

$$
s_{k}=T\left(r_{k}\right)=\sum_{j=1}^{k} p_{r}\left(r_{j}\right)=\sum_{j=1}^{k} \frac{n_{j}}{n}
$$

The obtained intermediate output will not be in uniform manner. But it will be in noticeable dynamic range.

\section{Convolutional neural networks and tracking the} object

Ideally the object is received in the input frame and input is directed towards the sizer which is pixel by pixel value counter by using histogram values and then the values are copied and then foreground and the background and the contrast, tint, tone of the image are gathered. Then the images are run into the platform of $\mathrm{CNN}$ which is the collection of various types of data set which are embedded in the cloud or the local platform. The local platform which carries the intention with collecting data should possess a huge memory for storage of images information for further processing.

The source code has a particular language i.e., Python and the $\mathrm{CNN}$ must run in parallel so that the informations are brought together via a separate intermediate code.

After detecting the image and extracting from the background noise further process are initiated. The extraction of texts is one of the process intended below.

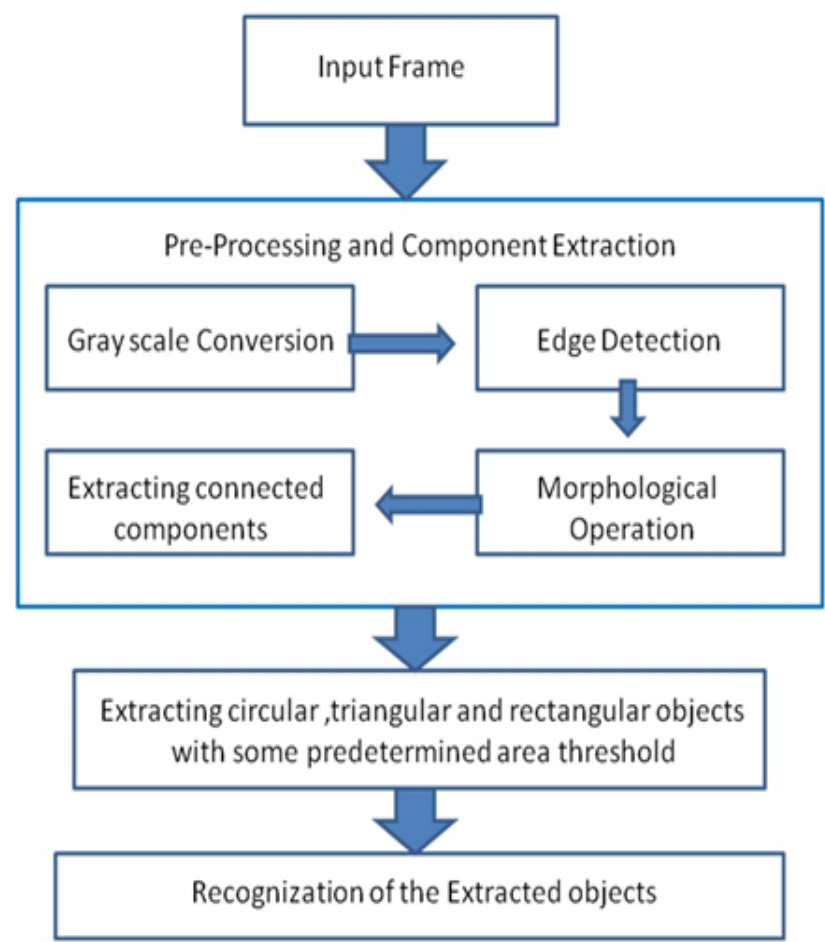

\section{EXTRACTION OF TEXTS \& RESULTS}

\section{A. Methodologies}

The process of detecting the texts is grouped under major level 1.Detection stage and 2.Recognition stage.

- Under detection stage the Localization and Verification are embedded.

- Under recognition stage the Segmentation and Recognition are done. 
They are generally classified into stepwise methods or integrated methods. They also have processes through detection, classification, segmentation and recognition.

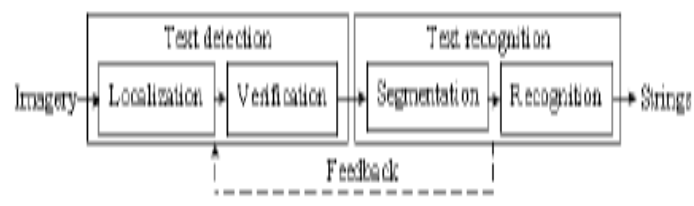

(a)

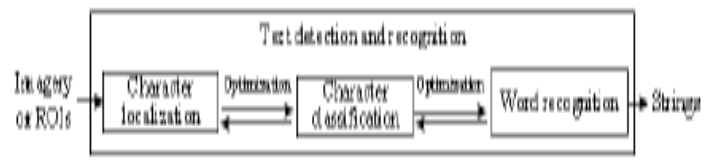

(b)

\section{B. Methods of stepwise}

Stepwise strategies pursue phases of text detection and localization, classification, recognition, and acknowledgment and expel background part from the value in each stage. Since they are measures in lexicon size, they can be utilized to perceive texts from pictures of the volume of content. For text recognition achieved by utilizing convolutional neural systems based classifier. Text detection, following, character division, acknowledgment, and remedy are essential handling steps utilized in this methodology. Neural systems based even text identification is performed, trailed by measurable horizontal based calculation for character division. Convolutional neural grouping is utilized for acknowledgment alongside language. Likewise pursues stepwise techniques to recognize writings of subjective introduction from normal pictures. Segment extraction, part investigation, applicant connecting, and chain examination are four phases through which proposed framework continues. Associated segments are separated in the segment extraction organize utilizing edge discovery pursued by SWT.

\section{Integrated Methodologies}

Integrated techniques centre on distinguishing specific words from pictures. Incorporated strategies frequently dodge segments organize or recognition it with word acknowledgment or integrating stage. These methods are utilized for applications with the little size of lexicon perceiving a fixed arrangement of words. Integrated strategy for word spotting from normal scenes the background. It utilizes character acknowledgment and word setup organizes in this framework. It crops locale around the content from a picture and uses it as an information picture alongside vocabulary for word acknowledgment. It utilizes Histogram of Oriented Gradients (HOG) highlights with the closest neighbour classifier for character location. Word acknowledgment speaks to each word in the dictionary in the type of chain of associated characters and matches it with the yield of character acknowledgment stage to get the closest word for a lot of characters.Performs start to finish content scene confinement and acknowledgment by keeping numerous divisions of single characters until the last phase of handling where character settings in content line are known. This framework recognizes the character as outside areas, for example, locales whose external limit pixels have higher qualities than the area. The framework utilizes threshold, adjacency, and shading spacecontrast values as three parameters for each recognized character and stores their different divisions from which ideal qualities are chosen dependent on settings of characters in the content line. Any single parameter does not ensure effective outcomes, which causes the proposition of putting away different divisions of three parameters. For arrangement determination process, content locales are partitioned into content lines utilizing thorough inquiry strategy, trailed by the dismissal of low certainty districts in content lines and development of guided diagram by doling out scores to every hub and edge from which right succession of characters is chosen.

\section{Text detection and localization}

Textdetecting manages distinguishing nearness of the content in the info picture while textlocalizationsays about the position of the forms and structures gatherings of text areas by wiping out limit of the background. textdetection and localization process is performed utilizing component analysis or locale based techniques.

Connected component (cc) examination strategy frames diagram of associated focuses dependent on shading or edge highlights from binarized picture, associated segment investigation is utilized to identify and limit content regions. Utilizes productive cc extraction strategies as opposed to utilizing cc separating approach. Extricates message in type of associated segments by applying pixel-put together requirements with respect to segments. Speaks to strategy for extraction of connected components.

Region based techniques isolate pictures into little locales utilizing windows and scan these areas for the nearness of content utilizing surface or morphological activities since content and non-content districts have distinctive literary properties. Utilizes $64 \times 32$ pixels window and applies Modest AdaBoost classifier on 16 distinctive spatial sizes of pictureto classify text and non-text considering large variations in text size. Unsupervised learning approach is used by [11], on small $8 \times 8$ grayscale patches of pictures for feature learning. It further uses $32 \times 32$ pixels of image for content extraction, text detection training and character classifier training.

\section{E. Classification of text extraction}

After text recognition and limitation organize yield may contain non-content text alongside content regions as false positives. Characterization stage confirms content locales and wipes out non-content regions utilizing order calculations. This stage can likewise be called as check. Characterization calculations are either administered or unsupervised. Directed calculations know properties of content, for example, shading, measure, surface, and so on before arrangement. Unsupervised calculations don't have earlier learning about content highlights. Directed 
characterization calculations need preparing before grouping. These calculations experience preparing to most likely concentrate highlights of the content to be ordered and utilize these highlights in the grouping stage. Utilizes a regulated learning approach. Limitations gion just as territory, stature and width requirements on square got in the discovery organize are utilized in [13] for characterization.

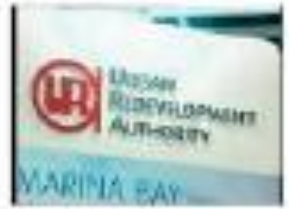

a)
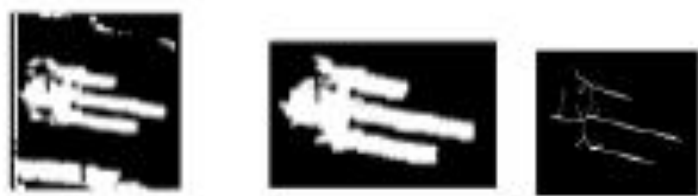

c)

d)

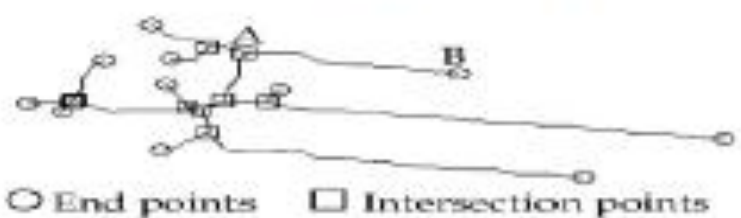

Unsupervised algorithms and texts attributives do not undergo extraction features and they will eject in proceeding algorithms. This adaptive wavelet transform which gives the results as successive approximation through LPF. Sub regions and indices are calculated.

\section{E. Text Segmentation}

Segmentation is process to separate the values of texts from background noise images and extract the bounded surface with help of algorithms from an image. Mainly binarization converts the gray scale image into black and white format to achieve good authentic segments with perspective of dark and bright variations.

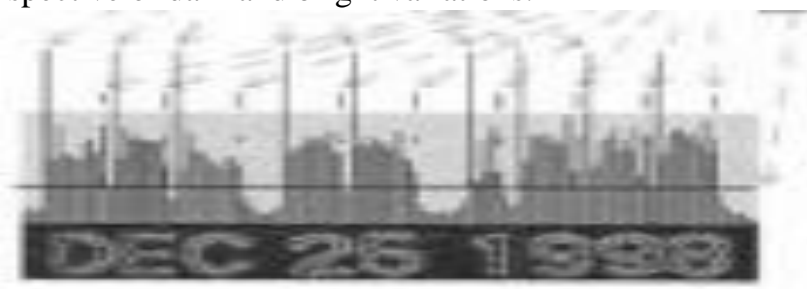

\section{G. Recognition of Texts}

Recognition is process of converting images of texts bounded from background into string of characters particular a word format. It divides images into multiple cuts outs into logical algorithms separations between methods.

Recognition mainly uses a OCR optical character recognition algorithm which makes initially are segmented into matrices supports and then connected components process it into gray scale unit to constraint module and feedback enabled.

\section{CONCLUSION}

This paper concludes the detecting an object and that object having a content of values which can be extracted by using the text detection and recognition algorithm, The process further proceeds with Object Detection and Usage of CNN then Text detection is further classified into Localization, classification of texts, segmentation of texts and recognition of texts and have been analysed its performance with theory.

\section{FUTURE ENHANCEMENT}

Many applications which runs on autopilot, automobiles and self-sustain drones with video surveillance, auto motion capturing camera and video recording are the future applicative advancements in image processing techniques. With the computer vision and machine learning which can be outsourced to these applications.

Mainly applicative consists of blinds immobility vehicle, unmanned aircrafts, video surveillance, and scanning objects with exact precision and text recognition.

\section{REFERENCES}

1. Text Detection and Recognition; A Review. Chaitanya R. Kulkarni, Ashwini B. Barbadekar-IRJET JUNE-2017

2. Real Time Bag Abandon Detection Using Open CVatulBorkar, Dr. Namgamode DHAVAL Pimplaskar. IJSER-NOV 2013.

3. Dr. S. NamgmodeAatulBorkar Moving object detection and tracking based on correlation transform wavelet in digital image-CIT JAN13.

4. Suresh and Dr. Mohan Abandoned objects detection in video surveillance camera in Internation conference on ICSIT DEC11

5. M.Bhagvya and Ms;. Ryoo and J.K.Agarwaal detection of abandon objects in crowded environment on IEEE07

6. K.Sumitha and P.Queslla detecting abandoned luggage items in public space CVPR 06.

7. P.L. VeemetianerZ.ZhangW.Yin stationary targe detection using the object video in public space IEEE 06

8. Qixiang Ye Text detection and recognition in imagery IEEE 2017

9. K.ELongui A comprehensive neural based approach for text recognition in videos using natural languages processing MR 11.

10. L. Nuemann J. matts on combining the segementation in scene text recognition IEEE 2013

11. An HTTP extension for secure transfer of confidential data International Journal of Engineering Development and Research, volume2, Issue 4. ISSN:2321-9939.

12. Preventing CSRF attacks International Journal of Advanced Research in Computer Science and Software Engineering (IJARCSSE), Volume 4, Issue 3, 2014.

13. A study of XSS worm propagation and detection mechanisms in online social networks by mohammadrezafaghani. rmeasures, 